\title{
Correlation between Left Ventricular End Diastolic Pressure and Torsion Dynamics in Patients with Diastolic Dysfunction, Speckle Tracking Imaging Study
}

\author{
Ahmed Emara*, Mahmoud Kamel, Said Shalaby, Ahmed Abd El-Razzak, \\ Wassam ELDin Hadad ELShafey \\ Department of Cardiology, Menoufia University, Menoufia City, Egypt \\ Email: ^am.emara@yahoo.com
}

How to cite this paper: Emara, A., Kamel, M., Shalaby, S., Abd El-Razzak, A. and ELShafey, W.E.H. (2019) Correlation between Left Ventricular End Diastolic Pressure and Torsion Dynamics in Patients with Diastolic Dysfunction, Speckle Tracking Imaging Study. World Journal of Cardiovascular Diseases, 9, 81-91.

https://doi.org/10.4236/wjcd.2019.92009

Received: January 14, 2019

Accepted: February 17, 2019

Published: February 20, 2019

Copyright $\odot 2019$ by author(s) and Scientific Research Publishing Inc. This work is licensed under the Creative Commons Attribution International License (CC BY 4.0)

http://creativecommons.org/licenses/by/4.0/

\section{(c) (i) Open Access}

\begin{abstract}
Background: Invasive measuring of LV end diastolic pressure (LVEDP) provides reliable assessment of $\mathrm{LV}$ diastolic dysfunction, but its invasive nature limits its use in daily practice. Accurate noninvasive assessment of LV diastolic dysfunction is highly desirable, and the relationship between the degree of LVEDP and LV torsion dynamics assessed by $2 \mathrm{D}$ speckle tracking is not clearly assessed; here in our study we aimed to assess the relation between the degree of LVEDP and torsion dynamics of left ventricle. Methods: The study included sixty patients divided equally into 3 groups according to the degree of LVEDP, group I: <12 mmHg, group II: $12-18 \mathrm{mmHg}$, and group III: >18 mmHg; complete conventional echo-Doppler study includes also annular septal $\mathrm{E}$ wave peak velocity, $\mathrm{E} / \mathrm{E}$ ' ratio and 2D-speckle tracking including parameters of LV global longitudinal strain (GLS), peak and time to peak of twist ratio (TR \& TT respectively), peak and time to peak of untwist ratio (UTR \& UTT respectively). Results: There was significant progressive decrease in GLS with progressive increase in LVEDP from I to III. Untwist ratio increased in group II and decreased in significant degree in group III; Twist ratio did not differ with different grades of LVEDP. LA diameter and E/E' increase from group I to III. The LVEDP is negatively correlated with the UTR and GLS is positively correlated with UTT, E/E \& LA diameter. The E/E' ratio is correlated negatively with the UTR, GLS and positively with LVEDP \& UTT and LA diameter. Conclusions: Noninvasive assessment of LV torsion and untwisting was feasible in patients with various grades of LVEDP and diastolic dysfunction; the peak untwist ratio increased in mild degree of increased LVEDP then decreased again with more increase in LVEDP; LVEDP
\end{abstract}


was negatively correlated with the peak untwist ratio and GLS was positively correlated with UTT and E/E'.

\section{Keywords}

Diastolic Dysfunction, LV End Diastolic Pressure, Torsion Dynamic

\section{Introduction}

Left ventricular diastolic dysfunction is a major cause of heart failure [1]. Invasive parameters of LV end diastolic pressure (LVEDP) and time constant of relaxation $(\tau)$ provide reliable assessment of LV diastolic dysfunction [2]; however, their routine use is limited due to procedural risks and high cost. Accurate noninvasive assessment of LV diastolic dysfunction is highly desirable. Echocardiography is usually used for noninvasive assessment and a number of echocardiographic parameters have been proposed for evaluating the diastolic dysfunction of LV [2] [3]. In patients with preserved LV ejection fraction (LVEF) the diagnostic accuracy of commonly used echocardiographic parameters is limited [3] [4] [5]. Torsion is an important spatial characteristic of LV mechanical function [6]. It results from the helical fiber arrangement of LV [7]. LV torsion from base to apex along a longitudinal axis is labeled as LV twist. LV twist is preserved or even increased in patients with diastolic dysfunction and normal systolic performance [8].

In this study we aimed to assess the correlation between invasively measured LVEDP degree and torsion dynamics of left ventricle in patients with diastolic dysfunction and preserved left ventricular systolic function.

\section{Patients and Methods}

The study included 60 patients planed for elective coronary angiography for suspecting CAD in the cath. lab of Menoufia university hospital from March 2016 to May 2017.

Patients were excluded if they had any of the following: significant coronary artery lesions by coronary angiography, patient with evidence of segmental wall motion abnormalities on echo exam, congestive heart failure, valvular heart disease, cardiomyopathy, renal disease, arrhythmias, congenital heart diseases, history of coronary bypass surgery, paced rhythm and abnormal QRS morphology and patients with poor echocardiographic windows.

All patients were subjected to informed written consent, complete history taking, and full physical examination, resting 12 leads ECG.

\subsection{Cardiac Catheterization}

Left ventricular catheterization was performed via the femoral approach, using 6 French sheaths. Left ventricular diastolic pressure was directly measured by fluid 
filled pigtail catheter attached to a pressure transducer. The Fourth intercostal spaces between the A-P diameters of the chest wall measured as Zero level. The left ventricular end diastolic pressure (LVEDP) was obtained by computer recording. Results from at least 5 beats were averaged. After that, standard technique coronary angiography was performed [9].

Included patients were classified into three groups according to LVEDP as follow:-

Group I: include 20 patients with normal LVEDD $<12 \mathrm{mmHg}$.

Group II: include 20 patients with mild increased LVEDD 12 - $18 \mathrm{mmHg}$.

Group III: include 20 patients with high elevated LVEDD $>18 \mathrm{mmHg}$.

\subsection{Standard Echocardiographic Examination}

Transthoracic echocardiographic examination was done using a commercially available echo machine (vivid E9, GE Medical Systems, Milwaukee,) according to the American Society of echocardiography recommendations [10]. Conventional Echocardiography measuring: left atrial, LV end diastolic and systolic dimensions, EF, Septal and Posterior wall thickness, All Doppler values represent the average of 3 consecutive beats. Mitral inflow was analyzed where E/A ratio and $\mathrm{E} / \mathrm{E}^{\prime}$ was computed.

\subsection{D Speckle Tracking Imaging Echocardiography}

Speckle tracking imaging (STI), module was used for assessment of rotational mechanics of LV through scanning and recording from left para-sternal short axis view of both basal and apical short axis planes to quantify basal and apical $\mathrm{LV}$ rotations using the same machine and probe with a probe frequency range $1.7-4 \mathrm{MHz}$ at a high frame rate (range $80-115$ frame/s). The basal level was marked as the plane showing the tips of mitral valve leaflets at its center with full-thickness myocardium surrounding the mitral valve. Then the transducer was positioned one or two inter-costal spaces more caudal and slightly lateral from the basal site to be perpendicular to the apical imaging plane [10] [11]. The apical level was defined just proximal to the level of LV apical luminal obliteration at the end-systole. The cross-section must be as circular as possible. We have to pay a careful attention to ensure that full thickness of myocardium is imaged throughout the cardiac cycle. Three consecutive cardiac cycles were digitally saved in a cine-loop format for later offline analysis. Offline analysis was done by an independent echocardiographer who was not involved in the image acquisition.

\subsection{Data Processing}

To analyze twist and untwist parameters, from the basal and apical short axis, a well-defined endocardial border and the regions of interest were adjusted to include all myocardial thickness without including the pericardium. The endocardial borders of both basal and short axis planes were manually traced 
and subsequently tracked by the software. If poor tracking quality was observed the region of interest was readjusted until acceptable tracking was obtained otherwise the segment with poor tracking was discarded and replaced by another one. After processing, curves of basal and apical LV rotation, twist, twist rate and untwist rate were automatically generated by the software. Twist was calculated as apical rotation relative to the basal rotation with counterclockwise rotation as viewed from LV apex expressed as positive value and clockwise rotation as a negative value. Peak LV twist, peak LV twist rate (as first positive peak after $\mathrm{R}$ wave on ECG), and peak LV untwist rate (as the first negative peak after aortic valve closure) were recorded. Cardiac cycle length was measured as R-R interval. Time to peak twist rate was measured as time from $\mathrm{R}$ wave to peak twist rate and time to peak untwist rate was measured as time from $\mathrm{R}$ wave to peak untwist rate.

Statistical analysis:

Data were analyzed using SPSS software. Quantitative data expressed as mean and standard deviation Chi square test student $t$ test, Mann whiney $U$ test, Kruskal Walls test and correlation coefficient test.

\section{Results}

The study included 60 patients and were classified according to the degree of invasively measured LVEDP into three groups, no significant difference was found between the three groups as regards age, sex and prevalence of risks factors (Table 1).

There was non-significant difference as regards conventional echocardiographic

Table 1. Demographic criteria and risk factors of study population.

\begin{tabular}{|c|c|c|c|c|c|}
\hline & & $\begin{array}{c}\text { Group } 1 \\
20 \text { patients }\end{array}$ & $\begin{array}{c}\text { Group } 2 \\
20 \text { patients }\end{array}$ & $\begin{array}{c}\text { Group } 3 \\
20 \text { patients }\end{array}$ & $\mathrm{P}$-value \\
\hline Age & Mean \pm SD & $50.60 \pm 9.419$ & $53.82 \pm 7.387$ & $51.67 \pm 7.399$ & 0.62 \\
\hline \multirow{2}{*}{ Female } & NO & 6 & 8 & 4 & \multirow{4}{*}{0.73} \\
\hline & $\%$ within the group & $30.0 \%$ & $36.4 \%$ & $22.2 \%$ & \\
\hline \multirow{2}{*}{ Male } & NO & 14 & 14 & 14 & \\
\hline & $\%$ within the group & $70.0 \%$ & $63.6 \%$ & $77.8 \%$ & \\
\hline \multirow{2}{*}{$\mathrm{DM}$} & NO & 10 & 16 & 10 & \multirow{2}{*}{0.54} \\
\hline & $\%$ within the group & $50.0 \%$ & $72.7 \%$ & $55.6 \%$ & \\
\hline \multirow[b]{2}{*}{ HTN } & NO & 8 & 10 & 6 & \multirow[b]{2}{*}{0.85} \\
\hline & $\%$ within the group & $40.0 \%$ & $45.5 \%$ & $33.3 \%$ & \\
\hline \multirow{2}{*}{ Dyslipidemia } & No & 4 & 6 & 4 & \multirow{2}{*}{0.92} \\
\hline & $\%$ within the group & $20.0 \%$ & $27.3 \%$ & $22.2 \%$ & \\
\hline \multirow{2}{*}{ Smoking } & No & 5 & 7 & 6 & \multirow{2}{*}{0.65} \\
\hline & $\%$ within the group & $25 \%$ & $35 \%$ & $30 \%$ & \\
\hline
\end{tabular}

DM: Diabetes mulitas, HTN: Hypertension. 
parameters no significant difference as regards LVEDD, LVESD, and EF, there was progressive increase in the LA diameter and E/E' from group one to three, $\mathrm{E} / \mathrm{A}$ ratio is maximum in group III and lowest in group II (Table 2).

As regards to the speckle tracking echo, there were significant progressive decrease in GLS and untwist ratio with progressive increase in LVEDP from group I to III (Table 3, Figures 1-3).

Twist ratio did not differ with different grades of LVEDP.

While the twist time and untwist times were progressively prolonged from group I to III but in non-significant degree.

The LVEDP negatively correlated with the UTR and GLS while is positively correlated with UTT, E/A, E/E \& LA diameter. The E/E' ratio correlated negatively with the UTR, GLS and positively with LVEDP \& UTT and LA diameter. LA diameter correlated positively with LVEDP and E/E' and negatively with UTR (Table 4).

Table 2. Comparison between groups as regarding conventional echocardiographic parameters.

\begin{tabular}{cccccc}
\hline & & Group 1 & Group 2 & Group 3 & P-Value \\
\hline LVEDD (cm) & Mean \pm SD & $5.12 \pm 0.434$ & $4.99 \pm 0.476$ & $4.96 \pm 0.384$ & 0.56 \\
LVESD (cm) & Mean \pm SD & $3.360 \pm 0.2633$ & $3.191 \pm 0.3727$ & $3.278 \pm 0.3456$ & 0.74 \\
EF \% & Mean \pm SD & $63.10 \pm 4.771$ & $64.27 \pm 3.228$ & $63.11 \pm 3.551$ & 0.87 \\
EDV $(\mathrm{cm})^{3}$ & Mean \pm SD & $113.52 \pm 25.52$ & $111.8 \pm 21$ & $104.49 \pm 26.02$ & 0.13 \\
ESV $(\mathbf{c m})^{3}$ & Mean \pm SD & $39.64 \pm 11.62$ & $37 \pm 10.25$ & $35.37 \pm 11.48$ & 0.11 \\
LA diameter $(\mathrm{cm})$ & Mean \pm SD & $3.38 \pm 0.4 .2$ & $3.845 \pm 0.2 .58$ & $4.1 \pm 0.173$ & $<0.01$ \\
E/A ratio & Mean \pm SD & $1.05 \pm 0.32885$ & $0.65 \pm 0.61768$ & $2.34 \pm 0.31$ & $<0.01$ \\
E', cm/sec & Mean \pm SD & $8.1 \pm 0.03$ & $7.2 \pm 0.03$ & $4.7 \pm 0.05$ & 0.04 \\
E/E ratio & Mean \pm SD & $7.31 \pm 1.742$ & $9.64 \pm 1.64543$ & $11.70 \pm 2.07$ & $<0.01$ \\
\hline
\end{tabular}

LVEDD: left ventricular end diastolic diameter, LVESD: left ventricular end systolic diameter, EF: ejection fraction, EDV: end diastolic volume, ESV: end systolic volume.

Table 3. Comparison between groups as regarding speckle tracking data.

\begin{tabular}{|c|c|c|c|c|c|}
\hline & & Group 1 & Group 2 & Group 3 & P-Value \\
\hline $\begin{array}{l}\text { Global longitudinal } \\
\text { strain (GLS) \% }\end{array}$ & Mean \pm SD & $-20.76 \pm 1.6$ & $-19.5 \pm 1.41$ & $-12.4 \pm 1.9$ & $<0.01$ \\
\hline Apical rotation, degrees & Mean \pm SD & $9.81 \pm 4.62$ & $11.8 \pm 7.5$ & $8.9 \pm 2.5$ & 0.008 \\
\hline Basal rotation, degrees & Mean \pm SD & $-6.8 \pm 3.5$ & $-3.9 \pm 2.8$ & $-1.7 \pm 2.7$ & 0.05 \\
\hline $\begin{array}{l}\text { Peak twist ratio, } \\
\text { degrees/sec (TR) }\end{array}$ & Mean \pm SD & $92.08 \pm 17.595$ & $90.35 \pm 11.32$ & $91.43 \pm 16.119$ & 0.91 \\
\hline $\begin{array}{c}\text { Time to peak Twist } \\
\text { Msec (TT) }\end{array}$ & Mean \pm SD & $290.60 \pm 22.43$ & $302.18 \pm 26.99$ & $300.22 \pm 25.1$ & 0.42 \\
\hline $\begin{array}{l}\text { Peak untwist ratio, } \\
\text { degrees/sec (UTR) }\end{array}$ & Mean \pm SD & $-130.3 \pm 39.24$ & $-140.84 \pm 24.97$ & $-120.27 \pm 7.03$ & 0.04 \\
\hline $\begin{array}{c}\text { Time to peak Untwist } \\
\text { Msec (UTT) }\end{array}$ & Mean \pm SD & $561.90 \pm 65.65$ & $593.80 \pm 77.72$ & $618.70 \pm 16.6$ & 0.108 \\
\hline
\end{tabular}




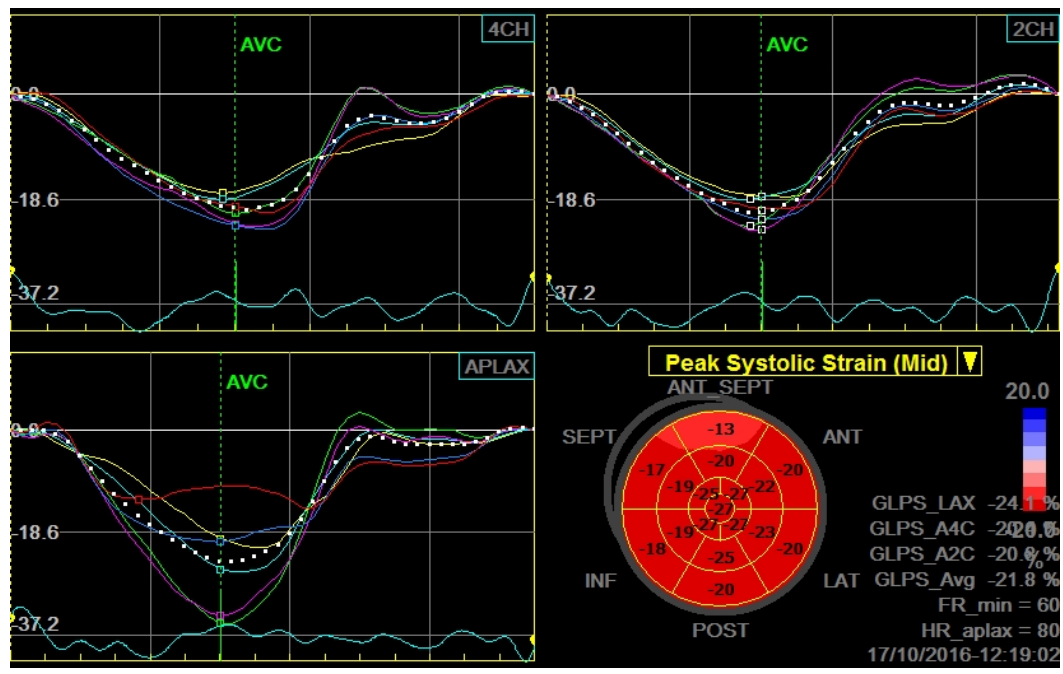

Figure 1. Longitudinal strain of LV of patient in group I.

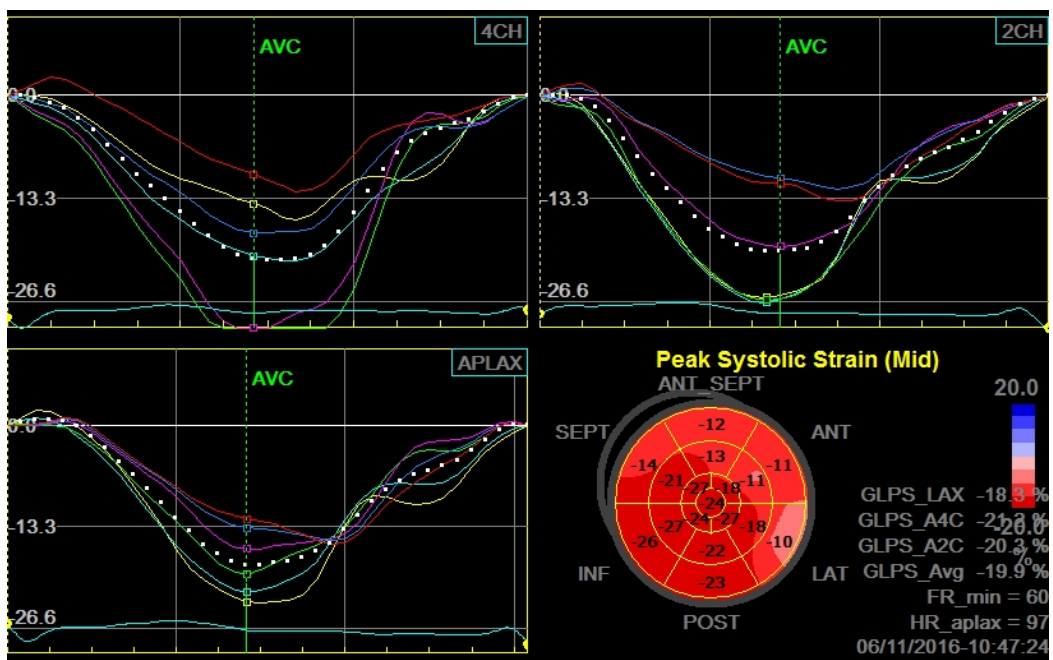

Figure 2. Example of LV longitudinal strain of patient in group II.

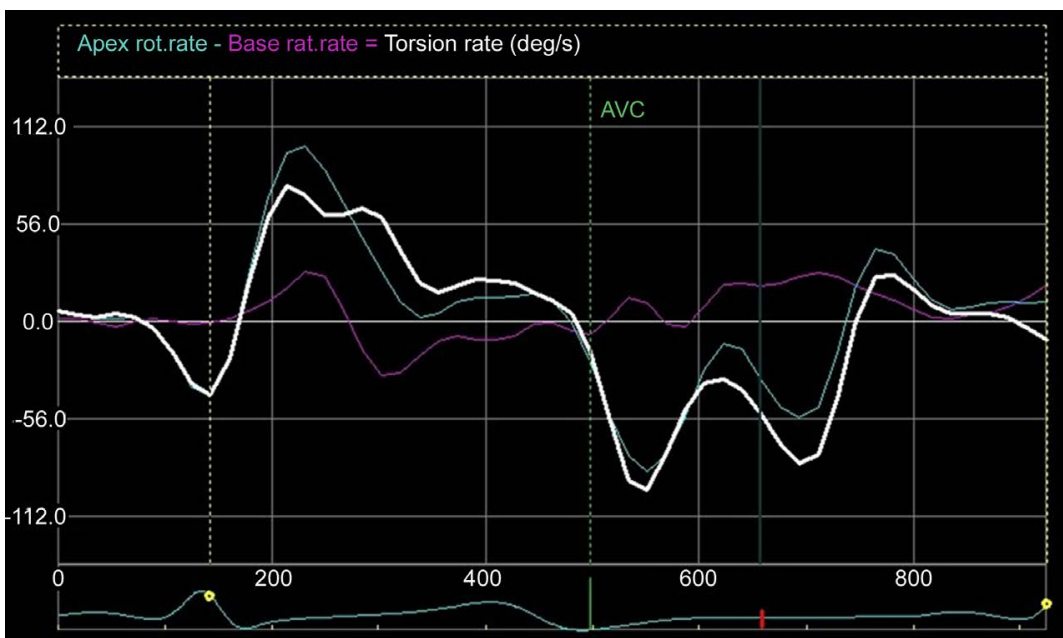

Figure 3. The positive wave before aortic valve closure is the peak twist ratio while the negative wave after aortic valve closure is the peak untwist ratio. 
Table 4. Correlation between untwist parameters and other parameters.

\begin{tabular}{|c|c|c|c|c|c|c|c|c|c|}
\hline & & LVEDP & LVEDD & LVESD & $\mathrm{EF}$ & LAD & $\mathrm{E} / \mathrm{A}$ & $\mathrm{E} / \mathrm{E}$ & GLS \\
\hline \multirow[b]{2}{*}{ UTT } & $\mathbf{R}$ & 0.511 & 0.116 & -0.009 & 0.264 & 0.550 & 0.294 & 0.525 & -0.106 \\
\hline & $\mathrm{p}$-value & $<0.01$ & 0.541 & 0.963 & 159 & $<0.01$ & 0.114 & $<0.01$ & 0.577 \\
\hline \multirow{2}{*}{ UTR } & $\mathbf{R}$ & -0.863 & 0.025 & 0.100 & -0.120 & -0.729 & -0.359 & -0.81 & 0.340 \\
\hline & p-value & $<0.01$ & 0.897 & 0.599 & 0.528 & $<0.01$ & 0.052 & $<0.01$ & 0.66 \\
\hline \multirow{2}{*}{ LVEDP } & $\mathbf{R}$ & & -0.185 & -0.188 & 0.096 & 0.754 & 0.608 & 0.928 & -0.607 \\
\hline & $\mathrm{p}$-value & & 0.327 & 0.320 & 0.615 & $<0.01$ & $<0.01$ & $<0.01$ & $<0.01$ \\
\hline \multirow{2}{*}{ GLS } & $\mathbf{R}$ & -0.607 & 0.206 & -0.015 & 0.250 & -0.344 & $-0.675^{\star *}$ & -0.502 & \\
\hline & p-value & $<0.01$ & 0.275 & 0.938 & 0.183 & 0.063 & $<0.01$ & $<0.01$ & \\
\hline \multirow{2}{*}{$\mathrm{TT}$} & $\mathbf{R}$ & 0.012 & -0.071 & -0.115 & -0.244 & 0.047 & -0.159 & 0.046 & -0.172 \\
\hline & p-value & 0.950 & 0.708 & 0.546 & 0.194 & 0.807 & 0.402 & 0.811 & 0.364 \\
\hline \multirow{2}{*}{ TR } & $\mathbf{R}$ & -0.012 & 0.171 & 0.289 & $-0.410^{*}$ & -0.125 & -0.097 & 0.055 & -0.242 \\
\hline & p-value & 0.949 & 0.368 & 0.122 & 0.024 & 0.511 & 0.609 & 0.772 & 0.197 \\
\hline \multirow{2}{*}{$\mathrm{E} / \mathrm{E}$} & $\mathbf{R}$ & 0.928 & -0.141 & -0.086 & 0.034 & 0.768 & $0.564^{* *}$ & & -0.502 \\
\hline & p-value & $<0.01$ & 0.456 & 0.651 & 0.856 & $<0.01$ & $<0.01$ & & $<0.01$ \\
\hline \multirow{2}{*}{ LAD } & $\mathbf{R}$ & 0.754 & $-0.376^{\star}$ & $-0.399^{*}$ & 0.192 & & $0.427^{\star}$ & 0.768 & -0.344 \\
\hline & $\mathrm{p}$-value & $<0.01$ & 0.041 & 0.029 & 0.310 & & 0.019 & $<0.01$ & 0.063 \\
\hline
\end{tabular}

UTT: untwist time, UTR: untwist ratio, TR: Peak twist ratio, LVEDP: left ventricular end diastolic pressure, GLS: global longitudinal strain, LAD: left atrium diameter.

\section{Discussion}

This study based on STE assessment of left ventricular torsion dynamic in patients with different degrees of LV diastolic pressure measured invasively. The major findings of this study were first: there were significant progressive decreases in GLS and untwist ratio with progressive increase in LVEDP from group I to III.

Second: apical rotation is increased from group I to II then decrease with group III. Third: the twist and untwist times were progressively prolonged from group I to III but in non-significant degree. Forth: The LVEDP negatively correlated with the UTR and GLS while is positively correlated with UTT, E/A, E/E \& LA diameter.

\subsection{Long-Axis Function in Patients with Diastolic Dysfunction}

In our study the longitudinal global strain was decreased progressively with increased LVEDP from group I to III.

Park et al. [12] found that patients with diastolic dysfunction and normal EF were found to have impaired systolic long-axis function compared with healthy controls. Their long-axis systolic velocity decreased progressively as diastolic dysfunction became worse. Long-axis Systolic function was decreased in all grades of diastolic dysfunction. 
However, LV torsion was increased in only mild diastolic dysfunction. This is further evidence that hyper-torsion and subsequent untwisting seem to be a compensatory mechanism for the reduced long-axis function of the myocardium to maintain normal LV filling.

\subsection{Left Ventricular Untwisting Ratio (UR) and Time to Untwisting Ratio}

In our study there were increase in untwist ratio and apical rotation from group one to group II then decrease again in group III significantly and there was progressive prolongation in time of UR but in non-significant degree

Wang et al. [13] found that patients with LV systolic dysfunction had a lower UR significantly in comparison with the control group. However, no significant differences existed between patients with diastolic dysfunction and the control group. In another study [14] including 75 patients proved to have diastolic dysfunction by Doppler mitral flow pattern and 25 normal volunteers. Included patients were classified into 3 groups: Group I: Including patients with Grade 1, abnormal relaxation pattern, (E/A ratio $<0.8)$. Group II: Including patients with Grade II or pseudo-normalized filling pattern $(0.8<\mathrm{E} / \mathrm{A}<1.5)$ Group III: Including patients with Grade III or restrictive filling pattern (E/A > 1.5). A significantly higher apical rotation, peak twist, peak twist ratio and untwist ratio among Group I (impaired relaxation), then these values decreased gradually from group I to group II and III, the Time to peak twist, twist ratio and untwist ratio showed a progressive non-significant difference between different grades of diastolic dysfunction.

Rebecaa et al. [15] found an increased untwist ratio in subjects with abnormal relaxation compared to those with pseudo-normal and restrictive pattern $(P$ $=0.001)$.

Masaaki Takeuchi et al. [16] study 49 patients with hypertension and according to the severity of $\mathrm{LVH}$, patients were divided into three groups [absence of LVH, mild LVH (LVMI: $116-131 \mathrm{~g} / \mathrm{m}^{2}$ in men and LVMI: $96-108 \mathrm{~g} / \mathrm{m}^{2}$ in women), and moderate-severe LVH (LVMI $\geq 132 \mathrm{~g} / \mathrm{m}^{2}$ in men and LVMI $\geq 109$ $\mathrm{g} / \mathrm{m}^{2}$ in women)], early diastolic untwisting and untwisting rates were reduced significantly in parallel to the LVH degree in hypertensive patients [16].

Several studies [17] [18] showed that by using tagged MRI diastolic untwisting is delayed and prolonged in patient with aortic stenosis. In AS patients, apical peak rotation is significantly increased. Thus, during diastole, an increase in untwisting velocity or a prolongation of untwisting is expected. In the pressure over-loaded hearts due to aortic stenosis, a tendency toward an increased untwisting velocity and a significant prolongation of untwisting duration into the filling phase of the LV is seen. Because of the prolongation of apical diastolic untwisting, there is an overlap of untwisting and filling.

\subsection{Correlation with LVEDP}

In our study LVEDP is negatively correlated with the UTR $(\mathrm{r}-0.863, \mathrm{P}<0.01)$ 
and GLS ( $\mathrm{r}=-0.607, \mathrm{P}<0.01)$ while is positively correlated with UTT $(\mathrm{r}=$ $0.511, \mathrm{P}<0.01)$, E/A $(\mathrm{r}=0.608, \mathrm{p}<0.01), \mathrm{E} / \mathrm{E}(\mathrm{r}=0.928, \mathrm{p}<0.01)$, LA diameter $(\mathrm{r}=0.754, \mathrm{p}<0.01)$.

Wang et al. [13] found that LV untwist ratio was significantly related to pulmonary capillary wedge pressure $(\mathrm{r}=-0.5, \mathrm{P}=0.05)$, LV end-diastolic volume $(\mathrm{r}$ $=-0.5, \mathrm{P}=0.05), \operatorname{LVESV}(\mathrm{r}=-0.8, \mathrm{P}=0.001)$ and $\operatorname{LVEF}(\mathrm{r}=0.6, \mathrm{P}=0.02)$.

In Masaaki Takeuchi et al. study [16] a weak but significant correlation was observed between the degree of untwisting or untwisting rates and $\mathrm{E} / \mathrm{A}$ ratio or isovolumic relaxation times.

Rebecca Perry et al. [19] stated that there is negative correlation between un twist rate and $\mathrm{E}$ and $\mathrm{E} / \mathrm{E}$ ' ratio.

The finding of our study can be explained as follow: In a normal heart, the onset of myofiber shortening occurs earlier in the endocardium than the epicardium. During pre-ejection, subendocardial shortening and sub-epicardial stretch contribute to a brief clockwise rotation of the LV apex and counterclockwise rotation of the base [8] [19]. During ejection, the activation and contraction of the sub-epicardial layer with larger radius of arm of momentum produces higher torque to dominate the direction of rotation, resulting in counter-clock wise of the apex and clockwise rotation the base leading to ventricular twisting. Twisting and shearing of the sub-endocardial fibers deform the matrix and result in storage of potential energy in the deformed intracellular giant protein titin filaments and in the extracellular collagen fibers to be converted into kinetic energy (restoring force) with the start of relaxation leading torecoil and untwist in early diastole [20]. In the majority of patients with diastolic dysfunction the subendocardial layer suffers first and becomes affected earlier leading to a decrease in its counterbalancing effect and the subepicardial domination of rotation becomes more prominent with increased both twist and untwist. With disease progression the sub-epicardial layer becomes affected leading to decrease of its domination with progressive decrease of both twist and untwist [21].

\section{Conclusion}

Noninvasive assessment of LV torsion and untwisting was feasible in patients with various grades of LVEDP and diastolic dysfunction; the peak untwist ratio increased in mild degree of increased LVEDP, then decreased again with more increase in LVEDP; LVEDP was negatively correlated with the peak untwist ratio and GLS was positively correlated with UTT and E/E'.

\section{Conflicts of Interest}

The authors declare no conflicts of interest regarding the publication of this paper.

\section{References}

[1] Kane, G.C., Karon, B.L., Mahoney, D.W., Redfield, M.M., Roger, V.L., Burnett, J.C., 
Jacobsen, S.J. and Rodeheffer, R.J. (2011) Progression of Left Ventricular Diastolic Dysfunction and Risk of Heart Failure. JAMA, 306, 856-863.

https://doi.org/10.1001/jama.2011.1201

[2] Paulus, W.J., Tschope, C., Sanderson, J.E., Rusconi, C., Flachskampf, F.A., Rademakers, F.E., Marino, P., Smiseth, O.A., De Keulenaer, G., Leite-Moreira, A.F., Borbely, A., Edes, I., Handoko, M.L., Heymans, S., Pezzali, N., Pieske, B., Dickstein, K., Fraser, A.G. and Brutsaert, D.L. (2007) How to Diagnose Diastolic Heart Failure: A Consensus Statement on the Diagnosis of Heart Failure with Normal Left Ventricular Ejection Fraction by the Heart Failure and Echocardiography Associations of the European Society of Cardiology. European Heart Journal, 28, 2539-2550. https://doi.org/10.1093/eurheartj/ehm037

[3] Nagueh, S.F., Smiseth, O.A., Appleton, C.P., Byrd, B.F., Dokainish, H., Edvardsen, T., Flachskampf, F.A., Gillebert, T.C., Klein, A.L., Lancellotti, P., Marino, P., Oh, J.K., Popescu, B.A. and Waggoner, A.D. (2016) Recommendations for the Evaluation of Left Ventricular Diastolic Function by Echocardiography: An Update from the American Society of Echocardiography and the European Association of Cardiovascular Imaging. Journal of the American Society of Echocardiography, 29, 277-314. https://doi.org/10.1016/j.echo.2016.01.011

[4] Santos, M., Rivero, J., McCullough, S.D., West, E., Opotowsky, A.R., Waxman, A.B., Systrom, D.M. and Shah, A.M. (2015) E/e' Ratio in Patients with Unexplained Dyspnea: Lack of Accuracy in Estimating Left Ventricular Filling Pressure. Circulation: Heart Failure, 8, 749-756.

https://doi.org/10.1161/CIRCHEARTFAILURE.115.002161

[5] Sharifov, O.F., Schiros, C.G., Aban, I., Denney, T.S. and Gupta, H. (2016) Diagnostic Accuracy of Tissue Doppler Index E/e' for Evaluating Left Ventricular Filling Pressure and Diastolic Dysfunction/Heart Failure with Preserved Ejection Fraction: A Systematic Review and Meta-Analysis. Journal of the American Heart Association, 5, e002530. https://doi.org/10.1161/JAHA.115.002530

[6] Omar, A.M., Vallabhajosyula, S. and Sengupta, P.P. (2015) Left Ventricular Twist and Torsion: Research Observations and Clinical Applications. Circulation: Cardiovascular Imaging, 8, e003029. https://doi.org/10.1161/CIRCIMAGING.115.003029

[7] Ahmed, M.I., Desai, R.V., Gaddam, K.K., Venkatesh, B.A., Agarwal, S., Inusah, S., Lloyd, S.G., Denney, T.S., Calhoun, D., Dell'italia, L.J. and Gupta, H. (2012) Relation of Torsion and Myocardial Strains to LV Ejection Fraction in Hypertension. JACC: Cardiovascular Imaging, 5, 273-281.

https://doi.org/10.1016/j.jcmg.2011.11.013

[8] Sengupta, P.P., Tajik, A.J., Chandrasekaran, K. and Khandheria, B.K. (2008) Twist Mechanics of the Left Ventricle: Principles and Application. JACC: Cardiovascular Imaging, 1, 366-376. https://doi.org/10.1016/j.jcmg.2008.02.006

[9] Yamamoto, K., Nishimura, R.A. and Redfield, M.M. (1996) Assessment of Mean Left Atrial Pressure from the Left Ventricular Pressure Tracing in Patients with Cardiomyopathies. American Journal of Cardiology, 78, 107-110. https://doi.org/10.1016/S0002-9149(96)00238-X

[10] Simpson, I.A. (1997) Echocardiographic Assessment of Long Axis Function: A Simple Solution to a Complex Problem? Heart, 78, 211-212. https://doi.org/10.1136/hrt.78.3.211

[11] van Dalen, B.M., Soliman, O.I.I., Kauer, F., Vletter, W.B., van der Zwaan, H.B., ten Cate, F.J. and Geleijnse, M.L. (2010) Alterations in Left Ventricular Untwisting with Ageing. Circulation Journal, 74, 101-108. https://doi.org/10.1253/circj.CJ-09-0436 
[12] Park, S.-J., Miyazaki, C., Bruce, C.J., Ommen, S., Miller, F.A. and Oh, J.K. (2008) Left Ventricular Torsion by Two-Dimensional Speckle Tracking Echocardiography in Patients with Diastolic Dysfunction and Normal Ejection Fraction. Journal of the American Society of Echocardiography, 21, 1129-1137. https://doi.org/10.1016/j.echo.2008.04.002

[13] Wang, J., Khoury, D.S., Yue, Y., Torre-Amione, G. and Nagueh, S.F. (2007) Left Ventricular Untwisting Rate by Speckle Tracking Echocardiography. Circulation, 116, 2580-2586. https://doi.org/10.1161/CIRCULATIONAHA.107.706770

[14] Ahmed, M.K., Soliman, M.A., Mena, M.B., Said, M. and Montaser, S. (2018) Relation of Diastolic Dysfunction to the LV Twist and Untwist Dynamic: Speckle Tracking Imaging Study. International Journal of Cardiovascular Research, 7, 4. https://doi.org/10.4172/2324-8602.1000362

[15] Perry, R., De Pasquale, C.G., Chew, D.P. and Joseph, M.X. (2008) Assessment of Early Diastolic Left Ventricular Function by Two-Dimensional Echocardiographic Speckle Tracking. European Journal of Echocardiography, 9, 791-795. https://doi.org/10.1093/ejechocard/jen148

[16] Takeuchi, M., Borden, W.B., Nakai, H., et al. (2007) Reduced and Delayed Untwisting of the Left Ventricle in Patients with Hypertension and Left Ventricular Hypertrophy: A Study Using Two Dimensional Speckle Tracking Imaging. European Heart Journal, 28, 2756-2762. https://doi.org/10.1093/eurheartj/ehm440

[17] Nagel, E., Stuber, M., Burkhard, B., Fischer, S., Scheidegger, M., Boesiger, P. and Hess, O.M. (2000) Cardiac Rotation and Relaxation in Patients with Aortic Valve Stenosis. European Heart Journal, 21, 582-589. https://doi.org/10.1053/euhj.1999.1736

[18] Stuber, M., Scheidegger, M., Fischer, S., Nagel, E., Steinemann, F., Hess, O. and Boesiger, P. (1999) Alterations in the Local Myocardial Motion Pattern in Patients Suffering from Pressure Overload Due to Aortic Stenosis. Circulation, 100, 361-368. https://doi.org/10.1161/01.CIR.100.4.361

[19] Ashikaga, H., van der Spoel, T.I., Coppola, B.A. and Omens, J.H. (2009) Transmural Myocardial Mechanics during Isovolumic Contraction. JACC: Cardiovascular Imaging, 2, 202-211. https://doi.org/10.1016/j.jcmg.2008.11.009

[20] Fukuda, N., Terui, T., Ishiwata, S. and Kurihara, S. (2010) Titin-Based Regulation of Diastolic and Systolic Functions of Mammalian Cardiac Muscle. Journal of Molecular and Cellular Cardiology, 48, 876-881. https://doi.org/10.1016/j.yjmcc.2009.11.013

[21] Nakatani, S. (2011) Left Ventricular Rotation and Twist: Why Should We Learn? Journal of Cardiovascular Ultrasound, 19, 1-6. https://doi.org/10.4250/jcu.2011.19.1.1 